\title{
A Rare Case Depicting MR Interpretation of Persistent Urachal Remnants in Adults
}

\author{
Ahluwalia VV', Rawat $A^{1}$, Narayan $S^{1}$, Gupta $A K^{2}$ \\ ${ }^{1}$ Department of Radiodiagnosis, Sarojini Naidu Medical College, Agra, Uttar Pradesh, India, \\ ${ }^{2}$ Department of Radiodiagnosis, U P Rural Institute of Medical Sciences \& Research, Saifai, \\ Etawah, Uttar Pradesh, India.
}

\begin{abstract}
Embryonic remnant of the cloaca and the allantois present after birth as a midline fibrous cord is usually detected in infancy and childhood. Failure to obliterate results in urachal disease is rare in adults. Varied remnants of the urachus have been reported. In this case report the usefulness of basic high resolution sonography for initial screening \& usefulness of MRI in delineating the length of tract in an adult has been highlighted.
\end{abstract}

Keywords: Pyoloroduodenal, Perforation, Sonography

\section{Introduction}

The urachus is a fibrous cord structure formed after the obliteration of the urogenital sinus and allantois during the embryologic process. Remnant of the urachus can present as patent urachus, vesicourachal diverticulum, urachal sinus or urachal cyst. In this case report the etiology, clinical presentation and MR imaging features are discussed.

\section{Case Report}

A 23 year old male patient presented with history of discharge from umbilicus and mild

Correspondence to: Dr. Anil Rawat, Junior Resident (M.D. Radiodiagnosis), Department of Radiodiagnosis, Sarojini Naidu medical College, Agra, Uttar Pradesh, India. Email: rawats20@gmail.com grade fever and itching around umbilicus for the past 5-6 months. He had not undergone any prior treatment.On physical examination, there was excoriation of skin and tenderness around umbilicus, but no palpable lump was detected.

\section{Imaging Features}

High resolution ultra-sonographic HRUS evaluation showed hypo echoic ill-defined collection in infraumbilical parities and a small sub-centimetre linear hypo echoic tract inferiorly.MR evaluation was done subsequently to confirm and outline the extent of tract pre-operatively. Thin sagittal $\mathrm{T} 2$ scanning on $0.5 \mathrm{~T}$ MR revealed long linear thin hyper intense tract extending from infraumbilical region till superior aspect of urinary bladder with corroborative axial imaging. 
No cystic out pouching, collection or surrounding soft tissue abnormality was seen. A diagnosis of persistent patent urachus was made.

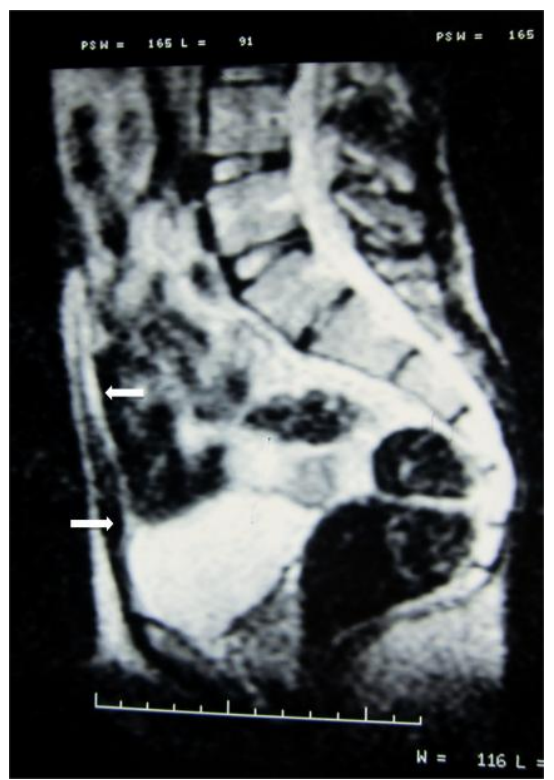

Fig 1: Thin sagittal $T 2$ image reveals a long linear hyper-intense tract extending from superior aspect of urinary bladder till umbilicus. There is no evidence of any branching, cystic out pouching, collection or surrounding soft tissue abnormality.

Subsequent surgery confirmed persistence of urachal remnant as a patent urachus and complete resection was done. Patient is asymptomatic on follow-up.

\section{Diagnosis}

Persistent urachal remnant (patent urachus).

\section{Discussion}

Embryonic remnant of the cloaca and the allantois present after birth as a midline fibrous cord is usually detected in infancy and childhood, Failure to obliterate results in urachal disease, However it is rare in
adults.Varied remnants of the urachus have been reported.

The congenital urachal anomalies are 3 times more common in males in comparison to females. ${ }^{1}$ There are 4 types anomalies including patent urachus (about $50 \%$ of cases), urachal cyst (about 30\%), urachal sinus (about 15\%), and urachal diverticulum (about5\%). ${ }^{2}$ Yiee et al. $^{3}$ reported that there were approximately two cases of urachal abnormality per 100,000 hospital admissions in adults.

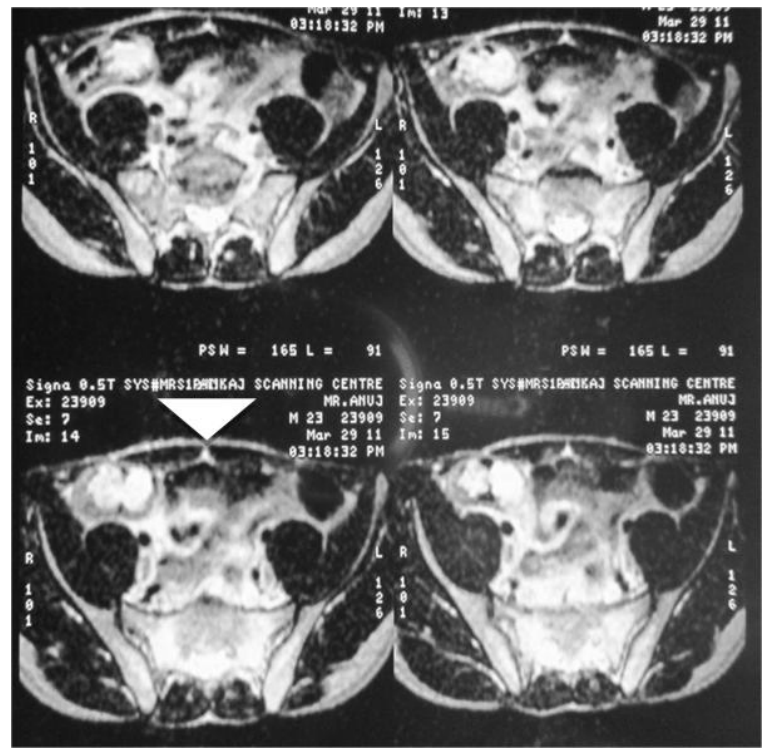

Fig 2: Thin axial T2 sections corroborate the fluid filled thin tract in superficial abdominal parieties.

The patient presents clinically with various symptoms such as umbilical discharge, low abdominal mass, tenderness with erythema, fever, urinary tract infection, haematuria, and peritonitis due to superadded infection of the urachal cyst. Infected urachal cyst can sometimes form urachal abscess that can results into septicemia. Due to severe urachal cyst infection, there can be formation of fistulous communication with bladder, bowel or umbilicus. Expansion of infected urachal cyst can cause rupture of abscess leading to 
acute abdomen that requires prompt surgical intervention. ${ }^{4}$

\section{Conclusion}

Urachal remnants are very rare in adults and the most common variants is patent urachus. Though the imaging was done at available low strength MR, its usefulness as noninvasive modality has been documented in comparison to poor/incomplete visualization on ultrasound or more tedious /invasive preoperative procedures like contrast studies/sinogram.

Hence, MRI should be recommended in suspected urachal remnant diseases preoperative road mapping.

Due to high recurrence rate and possibility of developing carcinoma in the urachal remnant, it is important to make sure of complete resection of the cyst wall throughout its course ${ }^{5}$, that may include removal of cuff of the bladder if there is communication between the urachal cyst and the bladder. Minimal invasive surgical approach is accepted as an alternative option in contrast to traditional open surgical approach because of faster recovery, less postoperative pain and better cosmetic results. ${ }^{6}$

\section{References}

1. Spataro RF, Davis RS, McLachlan MS, Linke CA, Barbaric ZL. Urachal abnormalities in the adult. Radiology 1983;149:659-63.

2. Mesrobian HG, Zacharias A, Balcom $\mathrm{AH}$, Cohen RD. Ten years of experience with isolated urachal anomalies in children. J Urol 1997;158(3 Pt 2):1316-8.

3. Yiee JH, Garcia N, Baker LA, Barber R, Snodgrass WT, Wilcox DT. A diagnostic algorithm for urachal anomalies. $\mathrm{J}$ Pediatr Urol 2007;3:500-4.

4. Goldman IL, Caldamone AA, Gauderer M, Hampel N, Wesselhoeft CW, Elder JS. Infected urachal cysts: a review of 10 cases. J Urol 1988;140:375-8.

5. Mesrobian HG, Zacharias A, Balcom $\mathrm{AH}$, Cohen RD. Ten years of experience with isolated urachal anomalies in children. J Urol 1997;158(3 Pt 2):1316-8.

6. Lee SI, Kim SS, Moon HY. Laparoscopic extraperitoneal resection of urachal cyst. J Korean Surg Soc 2010;78:267-9. 